\title{
TRAFFIC GENERATED BY A SEMI-MARKOV ADDITIVE PROCESS
}

\author{
J. BLOM AND M. MANDJES
}

\begin{abstract}
We consider a semi-Markov additive process $A(\cdot)$, i.e., a Markov additive process for which the sojourn times in the various states have general (rather than exponential) distributions. Letting the Lévy processes $X_{i}(\cdot)$, which describe the evolution of $A(\cdot)$ while the background process is in state $i$, be increasing, it is shown how double transforms of the type $\int_{0}^{\infty} e^{-q t} \mathbb{E}\left[e^{-\alpha A(t)}\right] \mathrm{d} t$ can be computed. It turns out that these follow, for given $\alpha \geq 0$ and $q>0$, from a system of linear equations, which has a unique positive solution. Several extensions are considered as well.
\end{abstract}

\section{INTRODUCTION}

Markov additive processes (MAPs) have proven an important modelling tool in communications networking [10, Ch. 6-7] as well as finance [3, 8], while nowadays also applications in biology are envisaged, see e.g. [7]. This has led to a vast body of literature; for an overview see for instance [2, Ch. XI]. A MAP is essentially a Lévy process whose Laplace exponent depends on the state of a (finite-state) Markovian background process; while this background process is in state $i$, the MAP, say $A(\cdot)$, evolves as a Lévy process $Y_{i}(\cdot)$ uniquely determined by its characteristic exponent $\psi_{i}(\cdot)$ [4]. MAPs can be considered as a non-trivial generalization of the standard Lévy process, and many results that were established earlier for Lévy processes were extended to the MAP framework. In particular, transforms of the type $\mathbb{E} \exp (-\alpha A(t))$, for given nonnegative $\alpha$ and $t$ can be given explicitly in terms of a matrix exponential [2, Prop. 2.1]; cf. also [9].

In a MAP it is implicit that the sojourn times in the states of the background process are exponentially distributed. Less is known about the situation in which this exponentiality assumption is lifted. To the best of our knowlegde, one of the few results known [5, Eqn. (2.1.3)] is for a very special case. The situation considered in [5] corresponds to a two-state background process, where one of the states (the 'off-state') corresponding to $A(\cdot)$ being constant, and the other state (the 'on-state') to $A(\cdot)$ growing with constant speed; the on-times are allowed to have a general distribution, but the off-times are still assumed to be exponentially distributed. The result is in terms of double transforms of the type $\int_{0}^{\infty} e^{-q t} \mathbb{E}\left[e^{-\alpha A(t)}\right] \mathrm{d} t$.

The goal of this note is to consider the situation of general sojourn times for all states of the background process, in which we could call $A(\cdot)$ a semi-Markov additive process (sMAP). We show that, for a given $\alpha \geq 0$ and $q>0$, the double transforms

$$
\int_{0}^{\infty} e^{-q t} \mathbb{E}_{i}\left[e^{-\alpha A(t)} 1_{\{X(t)=j\}}\right] \mathrm{d} t
$$

satisfy a linear system of equations; here $X(\cdot)$ records the state of the background process and $\mathbb{E}_{i}(\cdot)$ denotes expectation given that the background process starts off in state $i$ at time 0 . In addition, we show that this system of linear equations has a unique positive solution. Bearing the applications in, e.g., communications networking and biology in mind, we let the Lévy processes $X_{i}(\cdot)$ be increasing, but we also comment on what changes if we relax this assumption. 
Model. We proceed by presenting the formal model description of a semi-Markov additive process (sMAP). This $\operatorname{sMAP} A(\cdot)$ is defined as follows (where it is assumed that $A(0)=0$ ):

- Let $X_{n}$ be a discrete-time, irreducible Markov chain living on a finite state space $I:=$ $\{1, \ldots, N\}$. Its transition matrix is given by $P \equiv\left(p_{i j}\right)_{i, j=1}^{N}$, and the corresponding invariant distribution is $\varrho$. Without loss of generality we assume that $p_{i i}=0$.

- Let $\left(B_{i, n}\right)_{n \in \mathbb{N}}$ be, for any $i \in I$, a sequence of nonnegative i.i.d. random variables, distributed as the generic random variable $B_{i}$; the $N$ sequences $\left(B_{i, n}\right)_{n \in \mathbb{N}}$ are assumed to be mutually independent. Let $X(t)$ be a (continuous-time) semi-Markov chain on $I$, defined as follows. Supposing $X(0)=i$, the background process $X(\cdot)$ stays in $i$ for a period which is distributed as $B_{i}$. Then $X(t)$ jumps according to the transition matrix $P$ to some new state $j$. There it stays for a time distributed according to $B_{j}$, etc. It is assumed that $\mathbb{E} B_{i}$ is finite for all $i$.

- While in state $i, A(\cdot)$ evolves as an increasing Lévy process (also referred to as 'subordinator') $Y_{i}(t)$. Lévy processes are stochastic processes with stationary, independent increments, see e.g. [4] or [2, Ch. IX]. We denote the Laplace exponent of $Y_{i}(t)$ by $\varphi_{i}(\cdot)$ :

$\mathbb{E} e^{-\alpha Y_{i}(t)}=e^{-\varphi_{i}(\alpha) t}$.

The Lévy processes $Y_{i}(\cdot)$ are independent of the background process $X(\cdot)$.

We finish the model description by providing a number of examples of Lévy subordinators. An example used frequently is that of linear drifts; then $\varphi_{i}(\alpha)=\alpha r_{i}$, for $r_{i} \geq 0$. A second leading example is that of compound Poisson processes: while the background process is in state $i$, an i.i.d. sequence of jobs (distributed as a random variable $J_{i}$ ) arrives according to a Poisson process of rate $\lambda_{i}$, leading to

$$
\varphi_{i}(\alpha)=\lambda\left(1-\mathbb{E} e^{-\alpha J_{i}}\right) .
$$

For the case of jobs of size 1 , this reduces to $\varphi_{i}(\alpha)=\lambda_{i}\left(1-e^{-\alpha}\right)$. A last example relates to the record process of a Lévy process $Z(\cdot)$ that has negative jumps only (commonly referred to as 'spectrally negative'). Defining $T_{x}:=\inf \{t: Z(t)>x\}$, it is easily seen that the increasing process $T_{x}$ has stationary independent increments, and is therefore a Lévy process.

\section{ANALYSIS}

For ease, we start in our analysis by considering special increasing Lévy processes: while in state $i, A(\cdot)$ grows at a linear rate $r_{i} \geq 0$; later we consider the general case. In this 'linear drift case', we have

$$
A(t)=\int_{0}^{t} r_{X(t)} \mathrm{d} t .
$$

We are interested in the so-called double transform

$$
\mathscr{K}_{i j}(\alpha, q):=\int_{0}^{\infty} e^{-q t} \mathbb{E}_{i}\left[e^{-\alpha A(t)} 1_{\{X(t)=j\}}\right] \mathrm{d} t,
$$

where it is assumed that the background process has just jumped to state $i$ at time 0 (we come back to this issue in Remark 2.3). Interestingly, for $q>0$, this transform can be alternatively written as

$$
\frac{1}{q} \int_{0}^{\infty} q e^{-q t} \mathbb{E}_{i}\left[e^{-\alpha A(t)} 1_{\{X(t)=j\}}\right] \mathrm{d} t=\frac{\mathscr{L}_{i j}(\alpha, q)}{q}, \mathscr{L}_{i j}(\alpha, q):=\mathbb{E}_{i}\left[e^{-\alpha A\left(\tau_{q}\right)} 1_{\left\{X\left(\tau_{q}\right)=j\right\}}\right],
$$


where $\tau_{q}$ is an exponentially distributed random variable with mean $1 / q$; we call $\tau_{q}$ the 'killing epoch'. We first decompose

$$
\mathscr{L}_{i j}(\alpha, q)=\mathbb{E}_{i}\left[e^{-\alpha A\left(\tau_{q}\right)} 1_{\left\{X\left(\tau_{q}\right)=j, \tau_{q}<B_{i}\right\}}\right]+\mathbb{E}_{i}\left[e^{-\alpha A\left(\tau_{q}\right)} 1_{\left\{X\left(\tau_{q}\right)=j, \tau_{q} \geq B_{i}\right\}}\right] ;
$$

these terms we call $I_{1}$ and $I_{2}$, respectively.

First consider $I_{1}$. Then the killing epoch, $\tau_{q}$, takes place before $B_{i}$, i.e., the end of the sojourn time in state $i$. We thus obtain

$$
I_{1}=\int_{0}^{\infty} \int_{s}^{\infty} q e^{-s q} e^{-\alpha r_{i} s} f_{B_{i}}(t) 1_{\{i=j\}} \mathrm{d} t \mathrm{~d} s .
$$

Changing the order of integration, we eventually obtain

$$
I_{1}=1_{\{i=j\}} \cdot \frac{q}{q+\alpha r_{i}} \cdot\left(1-L_{i}\left(q+\alpha r_{i}\right)\right),
$$

where $L_{i}(\cdot)$ is the Laplace transform of $B_{i}$.

Now we consider $I_{2}$. Observe that if the sojourn time of state $i$ ends before the killing epoch, we can let the Markov chain jump, and sample the killing epoch again, due to the memoryless property. This reasoning leads to

$$
I_{2}=\int_{0}^{\infty} \int_{0}^{s} q e^{-s q} e^{-\alpha r_{i} t} f_{B_{i}}(t) \mathrm{d} t \mathrm{~d} s \cdot\left(\sum_{k \neq i} p_{i k} \mathscr{L}_{k j}(\alpha, q)\right) .
$$

Again interchanging the integrals, we obtain

$$
I_{2}=L_{i}\left(q+\alpha r_{i}\right) \cdot\left(\sum_{k \neq i} p_{i k} \mathscr{L}_{k j}(\alpha, q)\right) .
$$

We have thus arrived at the following result.

Theorem 2.1. Fix the final state $j$, and the values of $\alpha \geq 0$ and $q>0$. Then the vector

$$
x \equiv\left(\mathscr{L}_{1 j}(\alpha, q), \ldots, \mathscr{L}_{N j}(\alpha, q)\right)^{\mathrm{T}}
$$

is the unique solution of a system of equations $A x=b$. Here the entries of the matrix $A:=I-\tilde{P}$ are given by

$$
\tilde{P}_{i k}:=L_{i}\left(q+\alpha r_{i}\right) \cdot p_{i k},
$$

which is between 0 and 1 . In addition, the vector $b \equiv\left(b_{1}, \ldots, b_{N}\right)^{\mathrm{T}}$ is given by

$$
b_{i}:=1_{\{i=j\}} \cdot \frac{q}{q+\alpha r_{i}} \cdot\left(1-L_{i}\left(q+\alpha r_{i}\right)\right) .
$$

The uniqueness of the solution follows from the fact that $A$ is (strictly) diagonally dominant for $\alpha \geq 0$ and $q>0$, and hence invertible.

Corollary 2.2. Consider the above model, but now with the constant drifts (with slope $r_{i}$ ) replaced by Lévy subordinators $Y_{i}(\cdot)$ (with Laplace exponent $\varphi_{i}(\alpha)$ ). Then Thm. 2.1 goes through, with $q+\alpha r_{i}$ replaced by $q+\varphi_{i}(\alpha)$.

Remark 2.3. Above we assumed that the background process had just jumped to state $i$ at time 0 . In this remark we wish to compute the double transform when the background process starts off in equilibrium at time 0 . Assuming that the generic random variables $B_{i}$, with $i=1, \ldots, N$, have 
finite mean, it is clear that the long-run fraction of time that the background process $X(\cdot)$ spends in state $i$ is given by

$$
\pi_{i}:=\frac{\varrho_{i} \cdot \mathbb{E} B_{i}}{\sum_{j=1}^{N} \varrho_{j} \cdot \mathbb{E} B_{j}}
$$

Our goal is then to compute

$$
\mathscr{K}_{\pi, j}(\alpha, q):=\int_{0}^{\infty} e^{-q t} \mathbb{E}_{\pi}\left[e^{-\alpha A(t)} 1_{\{X(t)=j\}}\right] \mathrm{d} t,
$$

where the subscript ' $\pi$ ' denotes that we start off in equilibrium at time 0 . It is immediately clear that the state of the background process at time 0 is distributed according to $\pi$. It is important to note, however, that, supposing that this state is $i$, the time until the first jump is not distributed according to $B_{i}$, but according to it residual life time variant $B_{i}^{\star}$ :

$$
\mathbb{P}\left(B_{i}^{\star} \leq x\right)=\frac{1}{\mathbb{E} B_{i}} \int_{0}^{x} \mathbb{P}\left(B_{i}>y\right) \mathrm{d} y .
$$

Let $f_{B_{i}^{\star}}(\cdot)$ be the density of the residual life time, and $L_{i}^{\star}(\alpha)$ the corresponding Laplace transform:

$$
f_{B_{i}^{\star}}(x)=\frac{\mathbb{P}\left(B_{i}>x\right)}{\mathbb{E} B_{i}}, \quad L_{i}^{\star}(\alpha)=\frac{1-L_{i}(\alpha)}{\alpha \cdot \mathbb{E} B_{i}} .
$$

We obtain that, with $\mathscr{L}_{\pi, j}(\alpha, q):=q \cdot \mathscr{K}_{\pi, j}(\alpha, q)$,

$$
\mathscr{L}_{\pi, j}(\alpha, q):=\sum_{i=1}^{N} \pi_{i} \mathscr{L}_{i j}^{\star}(\alpha, q),
$$

where $\mathscr{L}_{i j}^{\star}(\alpha, q)$ equals

$$
1_{\{i=j\}} \cdot \frac{q}{q+\varphi_{i}(\alpha)} \cdot\left(1-L_{i}^{\star}\left(q+\varphi_{i}(\alpha)\right)\right)+L_{i}^{\star}\left(q+\varphi_{i}(\alpha)\right) \cdot\left(\sum_{k \neq i} p_{i k} \mathscr{L}_{k j}(\alpha, q)\right) ;
$$

note that the $\mathscr{L}_{i j}(\alpha, q)$ can be computed by using Thm. 2.1.

Example 2.4. We here consider a two-state sMAP, with $p_{11}=p_{22}=0$. Fix $j=1$; for reasons of symmetry the results for $j=2$ follow directly from those for $j=1$. Denote

$$
\zeta_{i}(\alpha, q)=L_{i}\left(q+\varphi_{i}(\alpha)\right), \quad \eta_{i}(\alpha, q):=\frac{q}{q+\varphi_{i}(\alpha)} .
$$

It follows that

$$
A^{-1}=\frac{1}{1-\zeta_{1}(\alpha, q) \cdot \zeta_{2}(\alpha, q)} \cdot\left(\begin{array}{cc}
1 & \zeta_{1}(\alpha, q) \\
\zeta_{2}(\alpha, q) & 1
\end{array}\right), \quad b=\left(\eta_{1}(\alpha, q) \cdot\left(1-\zeta_{1}(\alpha, q)\right), 0\right)^{\mathrm{T}} .
$$

Elementary computations yield that

$$
\left(\begin{array}{c}
\mathscr{L}_{11}(\alpha, q) \\
\mathscr{L}_{21}(\alpha, q)
\end{array}\right)=\frac{\eta_{1}(\alpha, q)\left(1-\zeta_{1}(\alpha, q)\right)}{1-\zeta_{1}(\alpha, q) \cdot \zeta_{2}(\alpha, q)}\left(\begin{array}{c}
1 \\
\zeta_{2}(\alpha, q)
\end{array}\right)
$$

After lengthy calculations it follows that, with $\xi_{i}(q):=1 /\left(q \mathbb{E} B_{i}\right)$ and suppressing the arguments of $\xi_{i}, \zeta_{i}$, and $\eta_{i}$,

$$
\begin{aligned}
& \hat{\mathscr{L}}_{11}(\alpha, q)=\eta_{1}-\xi_{1} \cdot \frac{\eta_{1}^{2}\left(1-\zeta_{1}\right)\left(1-\zeta_{2}\right)}{1-\zeta_{1} \zeta_{2}}, \\
& \hat{\mathscr{L}}_{21}(\alpha, q)=\xi_{2} \cdot \frac{\eta_{1} \eta_{2}\left(1-\zeta_{1}\right)\left(1-\zeta_{2}\right)}{1-\zeta_{1} \zeta_{2}} .
\end{aligned}
$$


Taking $\phi_{1}(\alpha)=0$ and $\phi_{2}(\alpha)=\alpha$ (so that we obtain an 'on-off source' that alternates between transmitting at a constant rate 1 and being silent), and assuming the off-times to have an exponential distribution, we indeed retrieve [5, Eqn. (2.1.3)].

\section{CONCLUDING REMARKS}

The results presented in this note are in terms of double transform, that, in general, cannot be inverted explicitly. Instead, one has to rely on numerical techniques to obtain accurate approximations for probabilities of the type $\mathbb{P}(A(t)>x)$. It is noted that recently, substantial progress has been made with respect to this type of inversion techniques. Besides the classical reference [1], we wish to draw attention on novel ideas developed by den Iseger, reported on in [6].

When the Lévy processes $Y_{i}(\cdot)$ are not necessarily subordinators, one clearly needs to work with characteristic exponents rather than Laplace exponents. One can easily derive the system of linear equations which is solved by the transform of the characteristic function of $A(t)$.

In many applications from practice (in particular those from biology), one considers the following situation. The process $X(\cdot)$ alternates between two states, say 1 and 2, and in state $i$ particles are generated according to a Poisson process with rate $\lambda_{i} \geq 0$. For general sojourn-time distributions, the double transform of $A(t)$ can be computed as described in this paper. One could, however, assume that every particle remains in the system for an exponential time (say with mean $1 / \mu$ ), and suppose that the goal is to find the distribution of the number of particles $N(t)$ that is present at time $t$. It is clear that the theory that we developed does not apply anymore; observe that the rate of particles leaving is proportional to the number of particles present (as in the $M / G / \infty$ queue). The analysis presented in this paper may serve as a first step towards finding the distribution of the number of particles $N(t)$ present at time $t$, or the corresponding steady-state distribution $N(\infty)$.

Acknowledgments. Part of this work was done while M. Mandjes was at Stanford University, Stanford, CA 94305, US. We are grateful to F. Bruggeman (CWI, Amsterdam, the Netherlands) and P. den Iseger (Cardano, Rotterdam, the Netherlands) for stimulating discussions.

\section{REFERENCES}

[1] J. AbATE and W. WhitT (1995). Numerical inversion of Laplace transforms of probability distributions. ORSA J. Comp., 7, 36-43.

[2] S. Asmussen (2003). Applied Probability and Queues, 2nd ed. Springer, Berlin.

[3] S. Asmussen, F. Avram, and M. Pistorius (2004). Russian and American put options under exponential phasetype Lévy models. Stoch. Process. Appl. 109, 79-111.

[4] J. Bertoin (1996). Léry Processes. Cambridge University Press, Cambridge, UK.

[5] J.W. CoHen (1974). Superimposed renewal processes and storage with gradual input. Stochastic Process. Appl. 2, 31-58.

[6] P. DEN IsEger (2006). Numerical transform inversion using Gaussian quadrature. Probab. Engg. Inf. Sci., 20, 1-44.

[7] M. DobrzyŃSKI and F. BRUgGeman (2009). Elongation dynamics shape bursty transcription and translation. Proc. Natl. Acad. Sci. USA, 106, 2583-2588.

[8] A. JoberT and L. Rogers (2006). Option pricing with Markov-modulated dynamics. SIAM J. Contr. Opt., 44, 20632078.

[9] G. KEsidis, J. WALRAND, and C.-S. CHANG (1993). Effective bandwidths for multiclass Markov fluids and other ATM sources. IEEE/ACM Trans. Netw., 1, 424-428.

[10] N.U. PRABHU (1998). Stochastic Storage Processes — queues, insurance risk, dams, and data communication. SpringerVerlag, New York, USA. 
CWI, Science PARK 123, 1098 XG Amsterdam, the Netherlands.

E-mail address: joke.blom@cwi.nl

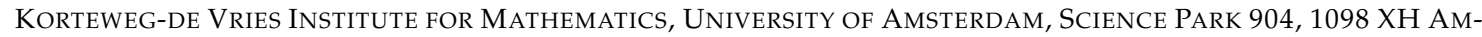
STERDAM, THE NETHERLANDS - EuRANDOM, EINDHOVEN, THE NETHERLANDS - CWI, AMSTERDAM, THE NETHERLANDS

E-mail address: m.r.h.mandjes@uva.nl 